\title{
Trials for second generation Covid-19 vaccines: Revisiting the debate over placebo use in developing country clinical trials
}

\section{PETER LURIE}

\begin{abstract}
This article compares the current debate over the use of placebos in developing country clinical trials of second generation Covid19 vaccines with the debates over previous paradigmatic cases raising similar issues. Compared to the earlier zidovudine and Surfaxin trials, Covid-19 vaccine trials are likely to confer lower risk to placebo groups and to offer a greater number and variety of alternative study designs. However, turning to the developing world to conduct studies that would be unacceptable in developed countries, simply on the ground that Covid-19 vaccines are generally unavailable in developing countries, is not ethically justifiable. This is so whether the justification is rooted in total absence of vaccine in a given country or in developing country vaccine prioritisation practices, because at root both derive from economic, not scientific conditions. However, the advent of variants that may create genuine uncertainty as to comparator vaccine effectiveness could justify a placebo control, depending on vaccine characteristics, variant prevalence, the degree of variant resistance, and the acceptability of immune-bridging studies. These factors must be considered together in the necessary case-by-case assessment of the ethical justification for any proposed trial.
\end{abstract}

Key words: Covid-19, vaccine trial, ethics, placebo, developing countries

We have arrived at a critical moment in the evolution of the Covid-19 pandemic: 13 vaccines have been authorised in at least one country, typically on the basis of randomised, placebo-controlled trials (1). Although not all of these products have received full regulatory approval, the high degrees of efficacy demonstrated - as high as $95 \%$ for some vaccines

Author: Peter Lurie (plurie@cspinet.org), Center for Science in the Public Interest, 1220 L Street, NW, Suite 300, Washington, DC 20005, USA.

To cite: Lurie P. Trials for second generation Covid-19 vaccines: Revisiting the debate over placebo use in developing country clinical trials. Indian J Med Ethics. 2021 Apr-Jun; 6(2) NS: 113-117.DOI: 10.20529/IJME.2021.023

Published online first on March 23, 2021.

Manuscript Editors: Ruth Macklin, Amar Jesani.

Peer reviewer: Rieke van der Graaf

(c) Indian Journal of Medical Ethics 2021
$(2,3)$ - and their general safety suggest that such approval is likely. We are therefore no longer in a period where the use of a placebo in future vaccine trials can be justified simply on the grounds that no effective product exists.

Against this backdrop, a World Health Organization (WHO) Ad Hoc Expert Group recently considered the use of placebos in the evaluation of Covid-19 vaccines, concluding that "Countries with limited or no access to a known effective vaccine could thus ethically permit placebo-controlled trials of vaccines of potential relevance to them even if effective vaccines were already being marketed elsewhere."(4) No ethical justification was provided.

To a large extent, the debate over the use of placebo controls in developing-country clinical trials after the effectiveness of an intervention has already been demonstrated dates back to the controversy over their use in studies seeking to prevent the transmission of the human immunodeficiency virus (HIV) from infected pregnant women to their offspring $(5,6)$. In 1994, three years before the controversy broke, a placebo-controlled trial of the drug zidovudine in the United States and France demonstrated that an approximately US $\$ 1,000$ regimen could reduce HIV transmission by about two-thirds. In the 15 studies that formed the focus of the subsequent controversy, researchers sought to evaluate the efficacy of a more limited regimen of zidovudine, a different antiviral, or nonantimicrobial regimens in reducing HIV transmission, using placebos or other regimens not known to be effective as comparator arms.

Even as the zidovudine trials controversy was subsiding, another trial related to the use of placebos in Covid-19 vaccine trials was being proposed. The drug in question was Surfaxin, a synthetic surfactant produced by Discovery Laboratories, a US pharmaceutical concern. Surfactants are drugs administered to infants with neonatal Respiratory Distress Syndrome, a substantial cause of neonatal mortality worldwide. At the time of the proposed trial in 2000, dozens of randomised, controlled trials, including many with placebos, had led to US Food and Drug Administration (FDA) approval of four surfactants. Surfactants were described in the New England Journal of Medicine almost a decade prior to the proposed research as "without doubt the most thoroughly studied new therapy in neonatal care" (7), and a Cochrane meta-analysis of synthetic 
surfactants in 2000 calculated that they could reduce 28-day mortality by $34 \%$ compared to placebo and concluded that "further placebo controlled trials of synthetic surfactant are no longer warranted."(8) Yet Discovery Laboratories proposed conducting just such a trial in Bolivia and other countries to be named at a future date.

Some observers (not this author) saw an ethical distinction between these two situations, even though both involved prospective follow-up of patients not receiving optimal therapy due to a lack of access derived from economic circumstances. It was clear to many that the researchers in the zidovudine studies, who were primarily supported by developed-world research agencies and multilateral entities, were engaged in a good-faith effort to identify affordable therapies tailored to developing-world conditions. But the Surfaxin situation was more suspect to those observers because the company's profit motive was readily discernible (as would be the case for the companies producing Covid-19 vaccines). As the US National Bioethics Advisory Commission declared, referring to Surfaxin, "In studies of this kind involving a disease that is life-threatening and one for which an established, effective treatment is available - a placebo control is not permissible."(9)

The controversy over the zidovudine trials led to reconsideration of the World Medical Association's Declaration of Helsinki, which in its current iteration avers that placebos may be used after the identification of a proven intervention when "for compelling and scientifically sound methodological reasons the use of any intervention less effective than the best proven one, the use of placebo, or no intervention is necessary to determine the efficacy or safety of an intervention" and that patients in such trials "will not be subject to additional risks of serious or irreversible harm as a result of not receiving the best proven intervention."(10) The International Ethical Guidelines for Health-related Research Involving Humans, prepared by the Council for International Organizations of Medical Sciences (CIOMS), declares that "As a general rule, the research ethics committee must ensure that research participants in the control group of a trial of a diagnostic, therapeutic, or preventive intervention receive an established effective intervention,"(11), but provides for exceptions generally similar to those in the Declaration of Helsinki.

The question before us, then, is whether there are ethical grounds to justify the use of placebos in developing countries, even in the wake of proven effective Covid-19 vaccines. Do the limited exceptions to the prohibitions on placebo use when an effective intervention has been identified pertain here? Stated differently, if you are someone who opposed the use of placebos in the zidovudine or Surfaxin trials (as this author did), can you distinguish the current Covid-19 vaccine situation sufficiently from those earlier trials to justify placebo use today? (This assessment assumes that additional conditions necessary for the ethical conduct of clinical trials in developing countries are met: the research is relevant to host country needs and any vaccine proven effective will be made reasonably available to subjects in the placebo group and to local residents more generally.)

\section{Comparing the risks}

Any consideration of placebos should include an attempt to estimate the magnitude of the risk associated with their use. Some risks may be so minimal that a placebo may be justified even when effective therapies are available (eg, antihistamines for seasonal allergy). But estimating this risk is an inherently tricky undertaking in the setting of Covid-19, for the very nature of a pandemic includes zeniths and nadirs, as well as variability in per capita burden between regions. But we can estimate excess illness as a result of lack of vaccination using data from the early vaccine trials. Specifically, we can estimate the difference between the percentages of subjects experiencing an event (symptomatic Covid-19) in the placebo groups and those with the same experience in the vaccinated groups. For the Pfizer/BioNTech and Moderna vaccines, respectively, those differences were $0.84 \% 2$ and $1.24 \% 3$ over the two-month study durations, implying that one in 119.0 $\left([1 / 0.84]^{*} 100\right)$ and one in $80.6\left([1 / 1.24]^{*} 100\right)$ subjects in the placebo groups contracted a symptomatic infection with SARS-CoV-2 that would have been prevented had they been randomised instead to the vaccine under study. If we assume, for estimation purposes, that $1 \%$ of such infections are fatal, we can hypothesise that about one in 10,000 placebo group recipients in a putative future trial might experience a vaccinepreventable fatality. In fact, among the 32,398 placebo patients in the two trials referred to above, there was a single fatality judged to be a result of Covid-19.

How do these risks compare with those in the zidovudine and Surfaxin trials? In the original zidovudine study, the perinatal infection rates in the treated and placebo groups were $8.3 \%$ and $25.5 \%$, respectively, meaning that a newborn infant's life was saved one in 5.8 times $\left([1 /(25.5-8.3)]^{*} 100\right)$ the regimen was administered (12). This efficacy and cost-effectiveness is far greater than the vast majority of interventions in modern medicine. In the Surfaxin trial, it was estimated that for every 19.1 times a placebo was used in place of a known effective comparator, the life of one neonate would be lost (13). Clearly, while risk to subjects in the Covid-19 vaccine trials exists, it is considerably smaller than in the earlier trials.

\section{Availability of alternative study designs}

In the ethical analysis of any clinical trial design, it is critical to consider alternative designs to the randomised, placebocontrolled trial, which is generally considered to be the gold standard in such assessments. Such alternative designs must be able to answer a public health-relevant research question and also be feasible in the context in which the trial is to be conducted. To the extent that these conditions are not met, the argument for a placebo-controlled trial is strengthened.

In the zidovudine trials, an alternative design was a non- 
inferiority trial in which, instead of seeking to demonstrate superiority to a placebo, the investigators attempt to establish that a new candidate treatment is no more than a preset percentage (ie,"noninferiority margin") inferior to the accepted therapy. Indeed, in the zidovudine trials, one National Institutes of Health-funded study in Thailand compared three simpler zidovudine regimens to the full regimen, without resorting to a placebo (14). Under realistic assumptions (including that, to demonstrate non-inferiority, the new therapy could permit perinatal transmission in no more than $6 \%$ more pregnancies than the standard regimen), a non-inferiority study would have required $24 \%$ more patients than a placebo-controlled one, a modest difference and one likely to be insignificant in the context of the delivery of antiviral drugs in developing countries at the time (5).

The Surfaxin predicament suggested two alternative designs to a placebo-controlled trial. One option was a non-inferiority study against an approved surfactant; in fact, the sponsor planned to conduct just such a study in Europe. Alternatively, the company could have undertaken a superiority study which would have compared the efficacies of Surfaxin and an approved surfactant head-to-head to determine which was more effective. But the Food and Drug Administration (FDA) explained that "a superiority trial [of Surfaxin] versus an approved therapy presents a clinical efficacy hurdle that the sponsor deems too high for this drug."(13)

For Covid-19 vaccine trials, there may, in theory at least, be still more alternatives to a randomised, placebo-controlled trial. First, a new candidate vaccine could be compared to an authorised product to establish which is superior. This design, while providing clinically useful information, is impractical if the comparator is one of the vaccines currently authorised in the United States, as the high efficacy rates (about 95\%) lead to formidable sample sizes. And sample sizes surely matter in the context of an evolving pandemic taking more lives per unit time than perinatal HIV transmission in the 1990s, for example.

A second possibility is a non-inferiority trial, as discussed above for the zidovudine and Surfaxin trials. $\operatorname{FDA}(15)$ and WHO(16) guidances on criteria for success for a Covid-19 vaccine require that the candidate vaccine demonstrate at least $50 \%$ efficacy with a lower bound of $30 \%$ efficacy. These guidances, issued in June and November 2020, respectively, further stipulated that noninferiority margins compared to an effective vaccine (none had been authorised at the time) be no more than $10 \%$. But few anticipated the $95 \%$ efficacy demonstrated by the first two authorised US vaccines $(2,3)$. A compelling case can be made that non-inferiority margins considerably wider than $10 \%$ would be acceptable, particularly if the vaccine has desirable characteristics such as a better safety profile, a single-shot dosing regimen, or less onerous storage requirements. Indeed, the Oxford/AstraZeneca vaccine, with an overall efficacy of $70.4 \%$ (95\% Cl: 54.8-80.6\%) (17) has been authorised in about two dozen countries for some of those reasons. If the Pfizer/BioNTech or Moderna vaccines were used as the active control, a non-inferiority margin as wide as $30 \%$ or more could be contemplated (the vaccine would still be at least $65 \%$ effective). One assessment of a range of scenarios found that a non-inferiority trial may require two to three times as many person-years of follow-up as a placebo-controlled trial, which could be achieved by a combination of larger sample sizes and longer follow-up (18). Such a trial "may enable reliable randomized evaluations of efficacy and safety of experimental Covid-19 vaccines," the authors concluded.

Critically, however, non-inferiority trials depend upon the "constancy assumption" - that the efficacy of the comparator vaccine in its original placebo-controlled trial will be retained in the setting of the non-inferiority trial. This assumption was met in the zidovudine and Surfaxin cases, but is subject to question in the context of newly emerging variants (see below).

A third option would be a challenge trial, in which an infectious dose of SARS-CoV-2 is administered intranasally. This design raises a slew of obvious ethical concerns of its own (19), the consideration of which is beyond the scope of this article. The appeal of such a design is that the investigators do not have to wait for subjects to become infected in their daily lives (a rare event from an epidemiological perspective), greatly reducing sample size and, potentially, study duration. Assuming that a consistently infectious dose can be identified, such a trial could be conducted with or without a placebo control. A study to identify the infectious inoculum has recently been approved in Britain (20).

The fourth option is theoretical at present, but may loom large in the future. If researchers can identify "correlates of immunity" - markers of a protective immune response such as neutralising antibodies - these correlates, rather than incident cases themselves, could become the measures of vaccine effectiveness. Such trials would be markedly reduced in size and could even be conducted without a comparator in some instances. To date, no such correlates have been confirmed.

\section{Reconsidering product availability}

The assessment of product availability in the context of the zidovudine and Surfaxin trials was relatively straightforward. Most observers agreed that the lack of availability of the products was the result of a set of social and economic circumstances that long predated the trials. It was over the ethical implications of these circumstances that people parted ways. The failure to administer zidovudine was often justified by reference to what was termed the "standard of care argument": people in the countries where the trials were taking place, it was said, had no access to zidovudine in the general course of medical treatment and so withholding the drug in clinical trials was justified. (It is worth noting that wealthy individuals in poorer countries undoubtedly did have access to the drug and that international activism in the years 
immediately following the trials led to a dramatic expansion in antiviral therapy in developing countries.) Tellingly, the WHO Ad Hoc Expert Group on Covid-19 vaccines also makes reference to the local standard of care. Others, including this author, argued that, notwithstanding any local conditions that may threaten safety or effectiveness, there can be only one standard of care: that proven to be safe and effective in appropriately conducted clinical studies. When a person fails to receive that intervention, it should not be sugarcoated with a term like "standard of care," but should instead be recognised for what it is: substandard medical care, whatever the cause, no matter local clinicians' good-faith efforts. Researchers with ready access to known effective medications had an obligation to provide them, we argued, particularly when lives were at risk and alternative designs available.

In retrospect, the zidovudine and Surfaxin cases seem more straightforward in this respect. For, with Covid-19 vaccines, the inevitable shortages of vaccines associated with a newly authorised product for which demand is high are layered on top of longstanding disparities in which residents of developing world countries lack basic healthcare. Prioritising scarce vaccines for those in greatest need based on the assessments of public health officials is inherently ethical. On a related topic, I have elsewhere argued that clinical researchers operating in a scenario where vaccine is initially in short supply, may continue to follow subjects in blinded fashion even after any vaccine is authorised, as long as the subjects are not yet in a priority vaccination group (21). (I suggested a blinded crossover design in which the placebo patients receive the vaccine and vice versa, but the blinding of investigators and subjects is maintained.) But if the lack of availability of a vaccine is grounded more in the underlying economic predicament of developing countries than in scarcity based on the inability of manufacturers to satisfy demand for technical reasons, that is not ground for withholding a known-effective intervention in a well-funded clinical trial. Of course, disentangling technical from economic causes of shortages will be a challenge.

Longstanding global inequities are already reflected in premarket purchase commitments between mostly developed countries and leading vaccine makers. By mid-November 2020, wealthy countries comprising $14 \%$ of the global population had already reserved $51 \%$ of the vaccine doses (22), leaving little opportunity for lower- and middle-income countries to gain access to these potentially life-saving products. Consequently, even when developing countries finally begin to obtain vaccine, they will be forced to designate scarce supplies to high-priority populations, a prioritisation driven largely by the initial lack of vaccine availability, itself a product of economic forces. But recruiting developing-country subjects who are not yet prioritised for vaccination due to shortages created by economic conditions bears strong resemblance to the zidovudine and Surfaxin situations. As the US experience demonstrates, withholding effective therapy in clinical studies has the potential to affect vaccine uptake for decades to come (23).

\section{The impact of Covid-19 variants}

The first assessments of the effectiveness of candidate vaccines started enrolling in the Northern hemisphere summer of 2020 and continued over the next several months, a period that predated the recognition of variant forms of SARS-CoV-2, at least some of which appear to be more transmissible (24). In South Africa, a country in which the emerging B.1.351 variant was predominant, a placebocontrolled trial of the Oxford/AstraZeneca vaccine, a product for which effectiveness had previously been demonstrated in other countries (17), showed no evidence of protection against mild-moderate Covid-19 (vaccine efficacy $=21.9 \% ; 95 \% \mathrm{Cl}$ : $-49.9 \%, 59.8 \%)(25)$. Certainly, the continuing dissemination of potentially vaccine-resistant variants alters the ethical calculus. If there is indeed equipoise over whether any vaccines would be effective in a particular country, a placebo-controlled trial could be justified. But if even one vaccine is believed by the community of relevant experts to be effective, that vaccine must be offered to subjects. The continued dissemination of these variants will necessitate frequent reassessment of the ethical justification for any new or ongoing trial. For example, FDA has announced that a known effective vaccine that has been modified to address emerging variants could be evaluated by the agency on the basis of immunogenicity studies, rather than new clinical trials (26).

\section{Conclusion}

Comparisons to paradigmatic ethical controversies in international research can be helpful in assessing the ethics of developing-country Covid-19 vaccine trials now that safe and effective vaccines have been identified. Compared to the zidovudine and Surfaxin trials, Covid-19 vaccine trials are likely to confer lower risk to placebo groups and to offer a greater number and variety of alternative study designs. It is noteworthy that in general there is no particular reason studies of these alternative designs could not be conducted in developed countries.

The argument that mere lack of vaccine availability justifies placebo use is unconvincing, whether that scarcity is the result of total absence of vaccine in a given country or in developing country vaccine prioritisation practices, because at root both derive from economic, not scientific conditions. However, the advent of variants that may create genuine uncertainty as to comparator vaccine effectiveness could justify a placebo control, depending on vaccine characteristics, variant prevalence, the degree of variant resistance, and the acceptability of immune-bridging studies.

These factors must be considered together in the necessary case-by-case assessment of the ethical justification for any proposed trial. 


\section{References}

1. Zimmer C, Corum J, Wee S-L. Coronavirus Vaccine Tracker New York New York Times. Updated 2021 Feb [cited 2021 Mar 21]. Available from: https://www.nytimes.com/interactive/2020/science/coronavirusvaccine-tracker.html.

2. Polack FP, Thomas SJ, Kitchin N, et al. Safety and efficacy of the BNT162b2 mRNA Covid-19 vaccine. N Engl J Med. 2020;383:2603-15.

3. Baden LR, El Sahly HM, Essink B, Kotloff K, Frey S Novak R, et al. Efficacy and safety of the mRNA-1273 SARS-CoV-2 vaccine. N Engl J Med. 2021 Feb 4;384(5):403-16.

4. WHO Ad Hoc Expert Group on the Next Steps for Covid-19 Vaccine Evaluation. Placebo-Controlled Trials of Covid-19 Vaccines - Why We Still Need Them. N Engl J Med. 2021 Jan 14; 384(2):e2. Doi: 10.1056/ NEJMp2033538. Epub 2020 Dec 2.

5. Lurie P, Wolfe SM. Unethical trials of interventions to reduce perinatal transmission of the human immunodeficiency virus in developing countries. N Engl J Med. 1997 Sep 18;337:853-6.

6. Angell $M$. The ethics of clinical research in the Third World. $N$ Engl J Med. 1997 Sep 18;337(12):847-9.

7. Jobe AH. Pulmonary surfactant therapy. N Engl J Med. 1993 Mar 25;328(12):861-8.

8. Soll RF. Synthetic surfactant for respiratory distress syndrome in preterm infants. Cochrane Database Syst Rev. 2000; (2):CD001149. Doi: 10.1002/14651858.CD001149.

9. Shapiro HT, Meslin EM. Ethical issues in the design and conduct of clinical trials in developing countries. N Engl J Med. 2001 Jul 12; 345(2): 139-42. Doi: 10.1056/NEJM200107123450212.

10. World Medical Association. Declaration of Helsinki (amended 2013). Ethical principles for medical research involving human subjects. Fortaleza, Brazil: WMA; 2013 Oct [cited 2021 Feb 18]. Available from: https://www.wma.net/policies-post/wma-declaration-of-helsinkiethical-principles-for-medical-research-involving-human-subjects/

11. Council for International Organizations of Medical Sciences (CIOMS) and WHO. International Ethical Guidelines for Health-related Research Involving Humans. Geneva; CIOMS; 2016. Available at: https://cioms.ch/ wp-content/uploads/2017/01/WEB-CIOMS-EthicalGuidelines.pdf

12. Connor EM, Sperling RS, Gelber R, Kiselev P, Scott G, O'Sullivan MJ, et al. Reduction of maternal-infant transmission of Human Immunodeficiency Virus Type 1 with zidovudine treatment. $N$ Engl J Med. 1994 Nov 3; 331(18):1173-80.

13. Lurie P, Wolfe SM. The developing world as the "answer" to the dreams of pharmaceutical companies: the Surfaxin story. In: Lavery JV, Grady C, Wahl ER, Emanuel EJ, eds. Ethical Issues in International Biomedical Research. Oxford, UK: Oxford University Press; 2007. pp. 159-70.

14. Lallemant M, Jourdain G, Le Coeur S, Kim S, Koetsawang S, Comeau AM, et al. A trial of shortened zidovudine regimens to prevent mother-tochild transmission of human immunodeficiency virus Type 1.Perinatal HIV Prevention Trial (Thailand) Investigators. N Engl J Med. 2000 Oct 5; 343(14):982-91.
15. US Food and Drug Administration, Center for Biologics Evaluation and Research. Development and Licensure of Vaccines to Prevent COVID19. Guidance for Industry. June 2020[cited 2021 Feb 18]. Available at: https://www.fda.gov/media/139638/download

16. World Health Organization. Considerations for Evaluation of COVID 19 Vaccines. 2020 Nov 25 [cited 2021 Feb18]. Available from: https:// cdn.who.int/media/docs/default-source/in-vitro-diagnostics/covid19/ considerations-who-evaluation-of-covid-vaccine_v25_11_2020.pdf? sfvrsn=f14bc2b1_3\&download=true

17. Voysey M, Clemens SAC, Madhi SA, Weckx LY, Folegatti PM, Aley PK, et al. Safety and efficacy of the ChAdOx1 nCoV-19 vaccine (AZD1222) against SARS-CoV-2: an interim analysis of four randomised controlled trials in Brazil, South Africa, and the UK. Lancet. 2021 Jan 9;397( 10269): 99-111.

18. Fleming TR, Krause PR, Nason M, Longini IM, Henao-Restrepo AM. COVID-19 vaccine trials: the use of active controls and non-inferiority studies. Clinical Trials. 2021. Feb 3; 1740774520988244. Doi: $10.1177 / 1740774520988244$.

19. Kahn JP, Henry LM, Mastroianni AC, Chen WH, Macklin R. Opinion: For now, it's unethical to use human challenge studies for SARS-CoV-2 vaccine development. Proceedings of the National Academy of Sciences USA. 2020;117:28538-42.

20. Adam K. U.K. gets approval to infect healthy volunteers in world's first coronavirus "challenge trial." Washington Post. 2021 Feb 17[cited 2021 Feb 17]. Available from: https://www.washingtonpost.com/world/ europe/covid-challenge-trial-uk/2021/02/17/a785a80c-7106-11eb8651-6d3091eac63f story.html.

21. Lurie P. Testimony before US Food and Drug Administration Vaccine and Related Biologics Advisory Committee on Pfizer/BioNTech COVID19 vaccine, December 10, 2020. Available from: https://cspinet.org/ sites/default/files/Lurie_VRBPAC_Testimony_12-10-2020.pdf.

22. So AD, Woo J. Reserving coronavirus disease 2019 vaccines for global access: cross sectional analysis. BMJ. 2020 Dec 15;371:m4750.

23. Bajaj SS, Stanford FC. Beyond Tuskegee - vaccine distrust and everyday racism. N Engl J Med. 2021 Feb 4; 384(5):e12. doi: 10.1056/ NEJMpv2035827.

24. Volz E, Mishra S, Chand M, Barrett JC, Johnson R, Geidelberg L, et al. Transmission of SARS-CoV-2 Lineage B.1.1.7 in England: insights from linking epidemiological and genetic data. medRxiv.20249034 [Preprint]. 2021 Jan 04[cited 2021 Feb 18]. Available from: https:// doi.org/10.1101/2020.12.30.20249034.

25. Madhi SA, Baillie V, Cutland CL, Voysey M, Koen AL, Fairlie L, et al. Safety and efficacy of the ChAdOx1 nCoV-19 (AZD1222) Covid-19 vaccine against the B.1.351 variant in South Africa. Medrxiv [Preprint]. 2021:2021.02.10.21251247v1.

26. US Food and Drug Administration, Center for Biologics Evaluation and Research. Emergency Use Authorization for Vaccines to Prevent COVID-19. Guidance for Industry. 2021 Feb 22[cited 2021 Feb 23]. Available from: https://www.fda.gov/media/142749/download 